\title{
Design of Non-Uniformly Spaced Circular Arrays of Parasitic Dipoles for Lower Side Lobe Level with Maximum Directivity
}

\author{
Hemant Patidar ${ }^{1 *}$, Gautam Kumar Mahanti ${ }^{2}$ \\ ${ }^{1,2}$ Department of Electronics and Communication Engineering, \\ National Institute of Technology, Durgapur, India \\ E-mail: hemantpatidar08@gmail.com
}

\begin{abstract}
This paper presents a new approach for circular array of parasitic dipoles composed by one active dipole for reduction of side lobe level with maximum directivity including mutual coupling. The desired goal is obtained by changing the spacing between the parasitic elements and length of the parasitic elements while the position and length of driven element is fixed. In addition to it, reflection coefficient $(R C)$ of the driven element is kept closer to the specified value. Matlab based method of moment code is used to evaluate the performance of circular antenna designs generated by QPSO algorithm. Two examples are presented to show the effectiveness of this proposed approach.
\end{abstract}

\section{Introduction}

Equally spaced and uniformly excited antenna arrays [1-2] provide higher directivity but they usually undergo from high SLL. The use of asymmetric excitations and nonuniform element spacing allows for enhanced degree of freedom in design [3]. In recent years circular arrays have become more in demand over other array geometries because of circular arrays have the potential to perform the scan in all the directions without a significant change in the beam pattern and provides azimuth coverage of $360^{\circ}$ [4-11]. Many researchers presented approaches for reduction of side lobe level with fixed beam width in circular antenna arrays by changing the excitation amplitudes and spacing between the elements [4-10]. Synthesis of circular antenna arrays with low side lobe level under both no beam scanning and beam scanning conditions using amplitude only and amplitude spacing are detailed in [11]. However, this method is not cost effective and also ends up in more complexity of their feeding network. Literature reports [1213] different methods to optimize the spacing and length of antenna elements for linear and planar array of parasitic elements.

This paper provides an alternative method: the active dipole is placed at the centre and surrounded by parasitic dipoles and it is done for a circular array. The desired goal is obtained by changing the length of parasitic elements, spacing between the parasitic elements keeping the position and length of active element fixed. Current across the driven and parasitic elements are calculated using the method of

moment's method that considers mutual coupling between array elements. First of all, the radiated electric field is computed using the current distribution and the array structure and after that directivity and other array parameters are calculated.

Matlab based method of moments (MoM) [14-16] code is used for simulation and to evaluate the performance of the antenna designs using optimization process generated by quantum particle swarm optimization (QPSO) [17-18].

Here, we have used QPSO algorithm because it provides better results than other algorithms in many antenna design problems [19-22]. QPSO perform better than PSO and its different versions on well-known benchmark functions [1718]. QPSO provides better results than backtracking search algorithm (BSA) in linear antenna array failure correction to obtain the fixed value of side lobe level and VSWR [19]. Furthermore, it performs better in non-uniformly spaced linear antenna array problems using amplitude excitations and element positions than firefly algorithm (FA) [20]. QPSO also provides better results in synthesis of nonuniformly spaced linear array of unequal length parallel dipole antennas than PSO for impedance matching with low side lobe level and main lobe tilting including uniform null filling, generally used in broadcasting applications [21] and provides better results than FA and cuckoo search algorithm (CS) to achieve the low side lobe level with impedance matching [22].

\section{Major contribution of the proposed work}

In the introduced work, synthesis is done on a nonuniformly spaced circular array consists of unequal height parasitic elements for a multi-objective problem. The proposed technique is different from [4-11] in the sense that the authors here considered the length of parasitic elements and spacing between the parasitic elements as design variables to obtain the desired requirements keeping the position and length of active element fixed. Moreover, this technique is different from [4-11] in the sense that authors here considered real antennas including mutual coupling effect. In addition to it, coupling effect is also compensated 
by minimizing the reflection coefficient of the driven element to the specified value.

\section{Theory}

We are considering here two examples with different parasitic dipoles of circular array in $X-Y$ plane. Parasitic dipoles parallel to $Z$-axis in circular ring composed by one driven element placed at centre are considered. Now the farfield pattern is generated by changing the length of parasitic elements and spacing between the parasitic elements while the length and position of driven element is kept fixed as shown in Fig. 1. In fact, changing the spacing between parasitic elements and length of parasitic elements modifies the positions and lengths of parasitic elements and as a result, currents across both parasitic and driven elements are modified owing to the mutual coupling effect.

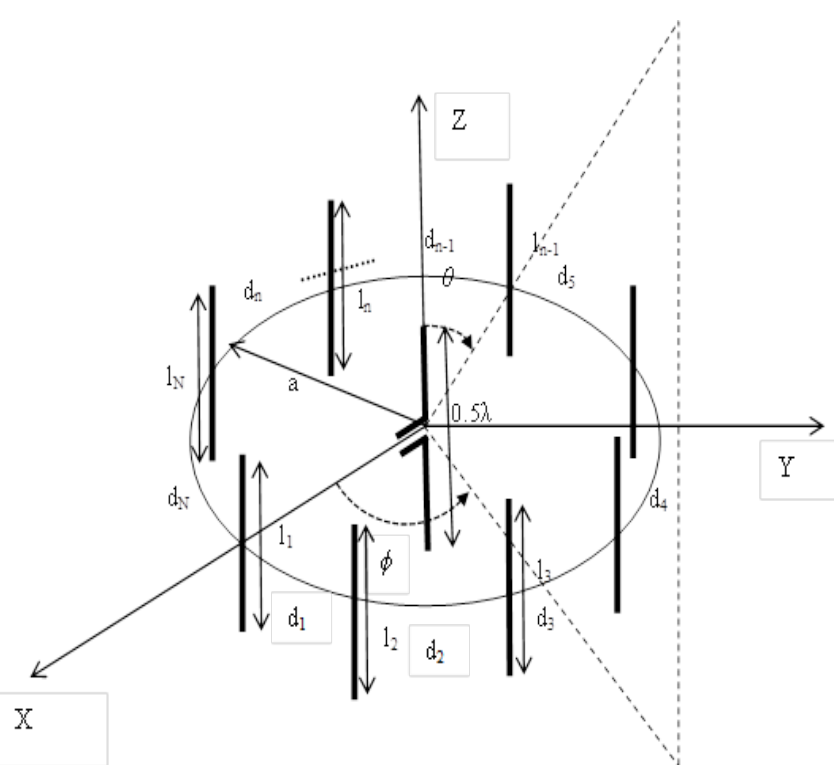

Fig. 1. Geometry of a circular array of parasitic dipoles with driven element placed at centre

Computation of far-field pattern requires evaluation of the current distribution. It is done by the matrix equation $[I]=[Z]^{-1}[V]$, where $[V]$ is the vector of voltages (known) applied to the driven and parasitic elements. The centre element is excited by unit voltage and voltages are kept zero for parasitic elements, [I] is the vector of complex current excitations of both the driven and parasitic elements, and $[Z]$ is the impedance matrix. The mutual impedance matrix depends on the geometry of array and its elements are calculated using [16]. The integration is solved using 32Point Gauss-Legendre quadrature integration formula.

Now the current distribution depends on both the length of parasitic elements and spacing between the parasitic elements and is required for calculation of radiation pattern.
The expression for the array factor $A F(\theta, \phi)$ of the considered circular array design incorporating the effects of mutual interactions is given by equation (1).

$$
A F(\theta, \phi)=\left[I_{0}+\sum_{n=1}^{N} I_{n} \exp ^{\left\{j k a \sin (\theta)\left[\cos \left(\phi-\phi_{n}\right)-\cos \left(\phi_{0}-\phi_{n}\right)\right]\right\}}\right]
$$

where $I_{0}$ is the complex current of the driven element, $I_{n}$ is the complex current of the $n^{\text {th }}$ parasitic element placed on circular ring with radius $a . N$ is the total number of parasitic elements. $\phi$ is the azimuth angle from $[-\pi, \pi], \phi_{0}$ is the angle of maximum radiation which is chosen as zero, $\phi_{n}$ is the angular position of $n^{\text {th }}$ parasitic element. $\theta=\pi / 2$, $k=2 \pi / \lambda=$ wave number, $\lambda=$ wavelength .

$$
\begin{gathered}
k a=2 \pi a / \lambda=\sum_{j=1}^{N} d_{j} \\
\phi_{n}=(2 \pi / k a) \sum_{j=1}^{n} d_{j}
\end{gathered}
$$

where $d_{n}$ is the distance from $n$ to $n+1$ element. The expression for $k a$ and $\phi_{n}$ are detailed in the articles [4-11]. The expression for the normalized gain of antenna array is detailed in [16], see chapter Hall'en Equations for Coupled Antennas [16, pp. 731].

In addition to it, minimization of reflection coefficient $(R C)$ of the driven element near to the specified value is also presented when the position and length of driven element are fixed. Assuming that the characteristic impedance $\left(Z_{o}\right)$ of the feed network is $50 \mathrm{ohm}$, the reflection coefficient $(R C)$ at the input of driven element is given by equation (4).

$$
R C=\left[\frac{\left|Z^{A}\right|-Z_{o}}{\left|Z^{A}\right|+Z_{o}}\right]
$$

where $Z^{A}$ is the active impedance of the driven element.

\section{Overview of QPSO Algorithm}

QPSO detailed in [17-18] is developed by Sun et al in 2004 is founded on the primordial law of particle swarm and rules of quantum mechanics in which all particles have the features of quantum deportment. It is described only by the position vector there is no velocity vector in QPSO. The protocol dealing with the movement of the particles in QPSO is quite different from that of standard PSO. In accordance with unpredictability theory of the quantum world, particles become visible at any position of search space with certain probability so the position and velocity of a particle cannot be found at the same instant. Flowchart of the QPSO algorithm is shown in Fig. 2. 


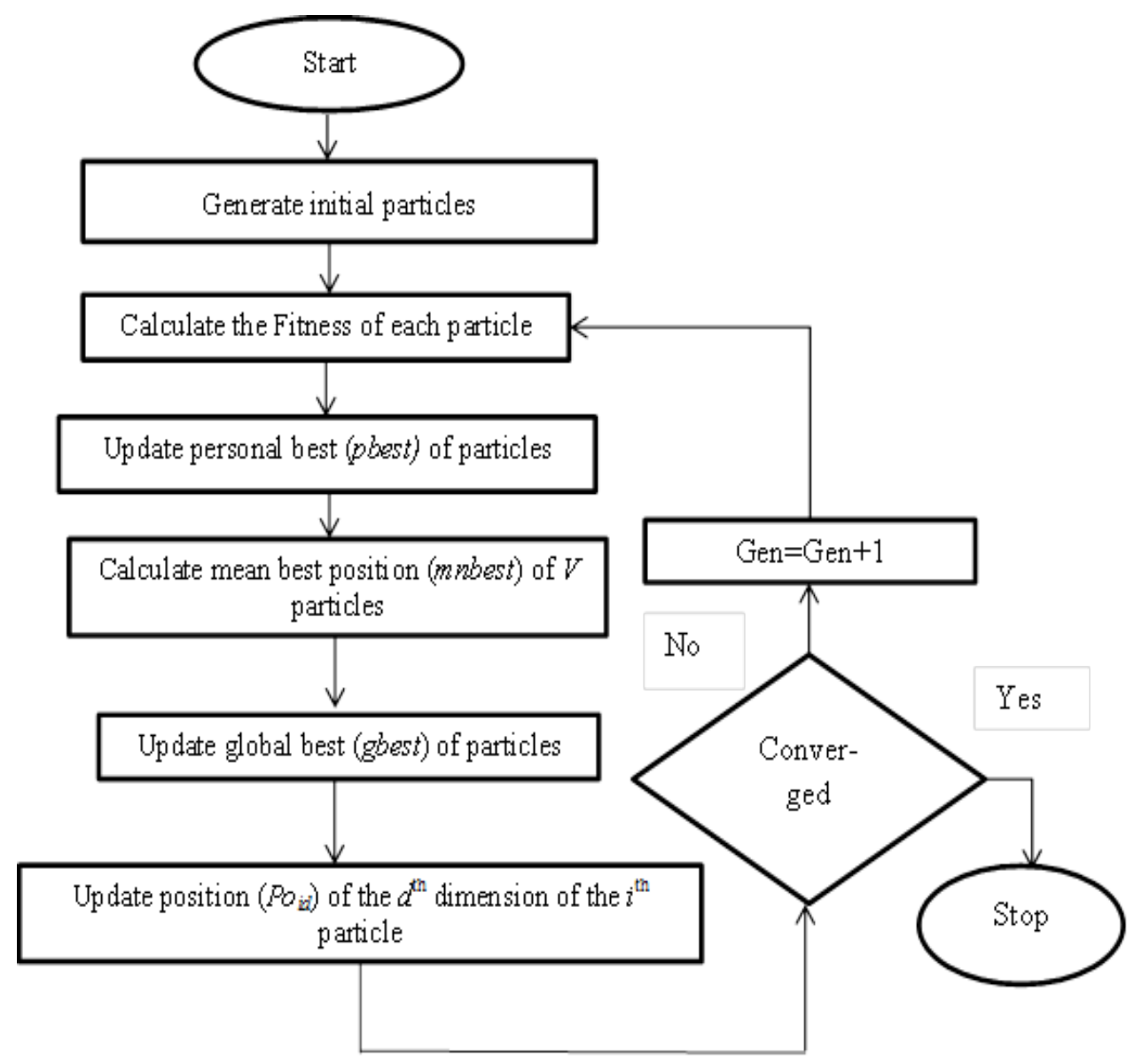

Fig. 2. Flow chart of QPSO algorithm

\section{Description of flowchart:}

1: Generate initial population of particles randomly between the minimum and the maximum operating limits in the Dsdimensional space.

2: Calculate the fitness value of each particle.

3: Update pbest of particles and its coordinates, if the present fitness value of the particle is superior to pbest of every particle, then allocate the present fitness value to pbest and allocate the present coordinates to pbest coordinates.

4: Mean best position (mnbest) of all $V$ particles is calculated by equation (5).

$$
\text { mnbest }=\frac{1}{V} \sum_{i=1}^{V} \text { pbest }_{i}
$$

The term mnbest is considered as the barycenter of all particles.

5: Update gbest of particles and its coordinates, if the present fitness value of the particle in the overall population is superior to global best (gbest).

6: The parameter $V L f$ is the local attractor of each $d^{\text {th }}$ dimension and $i^{\text {th }}$ particle and it is computed by equation (6).

$V L f_{i d}^{i t}=r n d 1_{i d}^{i t} *\left(\right.$ pbest $\left._{i d}\right)+\left(1-r n d 1_{i d}^{i t}\right) *($ gbest $)$

where $r n d 1$ is uniform random number between 0 and 1 . The term it refers to the current iteration.
7: Update position $\left(P o_{i d}\right)$ of the $d^{\text {th }}$ dimension and $i^{\text {th }}$ particle utilizing the following equations:

$P o_{i d}^{i t}=V L f_{i d}^{i t}+(-1)^{\operatorname{ceil}\left(0.5+r n d 2_{i d}^{i t}\right)} \times \alpha$

$\times \mid$ mnbest $-P o_{i d}^{i t-1} \mid \times \log _{e}\left(1 /\right.$ rnd $\left.3_{i d}^{i t}\right)$

If $P o_{i d}^{i t}<P o$ min , then

$P o_{i d}^{i t}=P o_{\min }^{d}+0.25 * r n d 4_{i d}^{i t} *\left(P o_{\max }^{d}-P o_{\min }^{d}\right)$

If $P o_{i d}^{i t}>P o_{\max }^{d}$, then

$P o_{i d}^{i t}=P o_{\max }^{d}-0.25 * r n d 5_{i d}^{i t} *\left(P o_{\max }^{d}-P o_{\min }^{d}\right)$

where $r n d 2, r n d 3$, rnd4 and $r n d 5$ are uniform random numbers between 0 and 1 . Equations (8) and (9) are applied along in each dimension within $\left(P o_{\max }^{d}, P o_{\min }^{d}\right)$ to clamp the position. These techniques are necessary to stop the particles from detonation if they try to come out of the required domain of interest. 
8: Repeat Steps from 2 to 7 till the maximum number of iterations being completed or when there is no further update of best fitness value.

It has one controlling parameter called contraction and expansion coefficient $\alpha$, which is used for controlling convergence speed and performance of the particle. In our case the value of $\alpha$ is 0.75 . Setting the value of $\alpha$ in the interval $(0.5,0.8)$, can generates good results, see literature report [17-18]. Details of the QPSO algorithm are available in the article [17-18].

\section{Numerical Examples}

In examples, 8 and 10 parasitic element arrays in circular rings are considered composed by one driven element placed at centre of the ring. Now keeping the position and length of driven element fixed, length of parasitic elements and spacing between the parasitic elements (the design variables) are found using QPSO. Number of current samples on the upper half of each element is 40. Method of moments with 'Exact' approximation is used for thin-wire kernel computation for Hellen's equation and 'Pulse' basis functions are used for solving Hellen's integral equation. In our case, QPSO is run for 200 iterations with a population (particle) size of 40 for both examples. For both the examples, length of driven element is fixed and it is $0.5 \lambda$. Radius of the dipoles is $0.003369 \lambda$.

Fitness function for generation of radiation pattern with desired goal is:

fitness $=\sum_{j=1}^{3} v t_{j} * F_{j}^{2}=\left[v t_{1} \times F_{1}^{2}+v t_{2} \times F_{2}^{2}+v t_{3} \times F_{3}^{2}\right]$ where

$F_{1}=\left\{\begin{array}{l}S L L_{o b}-S L L_{d e}, \text { if } \rightarrow S L L_{o b}>S L L_{d e} \\ 0, \text { if } \rightarrow S L L_{o b} \leq S L L_{d e}\end{array}\right.$

$F_{2}=\left\{\begin{array}{l}\operatorname{Dir}_{d e}-D_{i r_{o b}}, \text { if } \rightarrow D i r_{d e}>D i r_{o b} \\ 0, \text { if } \rightarrow D i r_{d e} \leq D i r_{o b}\end{array}\right.$

$F_{3}=\left\{\begin{array}{l}R C_{o b}-R C_{d e}, \text { if } \rightarrow R C_{o b}>R C_{d e} \\ 0, \text { if } \rightarrow R C_{o b} \leq R C_{d e}\end{array}\right.$

The coefficients $v t_{l}, v t_{2}$, and $v t_{3}$ are the relative weight specified to each term in equation (10). The choice of these weights denotes the importance of the concerned designed parameter associated with them.

The terms $S L L_{o b}$ and $S L L_{d e}$ are the obtained and the desired values of side lobe level, $D i r_{d e}$ and $D i r_{o b}$ are the desired and the obtained values of directivity and $R C_{o b}$ and $R C_{d e}$ are the obtained and the desired values of maximum reflection coefficient respectively. The aim is to obtain the desired values so the values of $F 1, F 2$, and $F 3$ are always the difference between obtained and desired values of antenna parameters until we didn't get the desired values. Once the aim is completed these values becomes zero in the fitness function which is our requirement to minimize the fitness function.

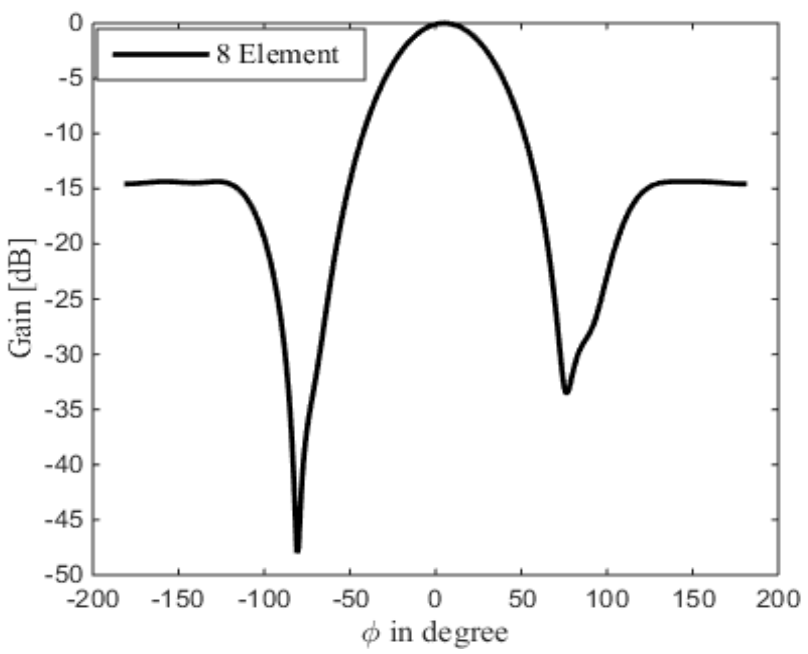

Fig. 3. H-pattern of circular array composed by one driven element for example1.

Desired value of Dir, SLL and $R C$ are $10 \mathrm{~dB},-15 \mathrm{~dB}$ and 0.33 for example1. The spacing between the array elements is varied from $0.10 \lambda$ to $1 \lambda$ and length of elements is varied from $0.40 \lambda$ to $0.70 \lambda$ to generate the radiation pattern with above requirements.Obtained $\mathrm{H}$-plane radiation pattern for example1 is depicted in Fig. 3.

Radiation pattern with side lobe level $(S L L)$ of $-14.35 \mathrm{~dB}$ and directivity of $9.1600 \mathrm{~dB}$ are obtained from simulation. In addition to it, reflection coefficient has been reduced to 0.4138 at the driven element. Table 1 shows the length and spacing for the 8 parasitic element array obtained from simulation.

Table 1: Length of parasitic elements and spacing between the parasitic elements

\begin{tabular}{cccc}
\hline & Length (1) & \multicolumn{2}{c}{ Spacing(d) } \\
\hline 11 & $0.5983 \lambda$ & $\mathrm{d} 1$ & $0.4880 \lambda$ \\
12 & $0.5847 \lambda$ & $\mathrm{d} 2$ & $0.3251 \lambda$ \\
13 & $0.5432 \lambda$ & $\mathrm{d} 3$ & $0.2710 \lambda$ \\
14 & $0.5300 \lambda$ & $\mathrm{d} 4$ & $0.1667 \lambda$ \\
15 & $0.5719 \lambda$ & $\mathrm{d} 5$ & $0.5325 \lambda$ \\
16 & $0.6804 \lambda$ & $\mathrm{d} 6$ & $0.3168 \lambda$ \\
17 & $0.6996 \lambda$ & $\mathrm{d} 7$ & $0.3417 \lambda$ \\
18 & $0.6998 \lambda$ & $\mathrm{d} 8$ & $0.4788 \lambda$ \\
\hline
\end{tabular}

It is assumed that $v t_{1}=8, v t_{2}=14$ and $v t_{3}=1$ for example 1 and $v t_{1}=5, v t_{2}=14$ and $v t_{3}=1$ for example 2. The parameter associated with more weights implies the importance of them over other parameters. 


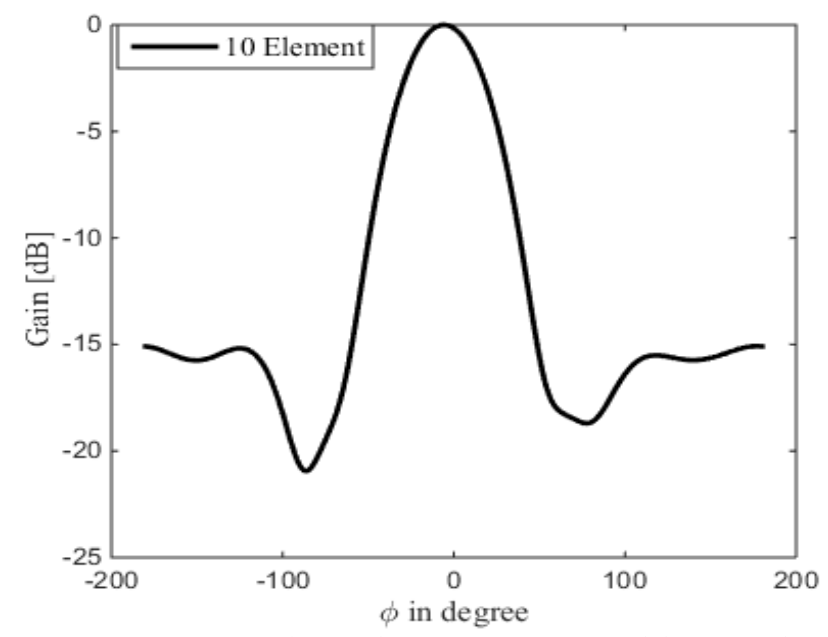

Fig. 4. H-pattern of circular array composed by one driven element for example2

Desired value of Dir, SLL and $R C$ are $12 \mathrm{~dB},-16 \mathrm{~dB}$ and 0.33 for example2. Here, the spacing between the array elements is varied from $0.10 \lambda$ to $0.80 \lambda$ and the length of elements is varied from $0.40 \lambda$ to $0.70 \lambda$ to generate the radiation pattern with above requirements. Obtained H-plane radiation pattern for example2 is shown in Fig. 4.

Radiation pattern with a side lobe level (SLL) of $-15.078 \mathrm{~dB}$ and a directivity of $9.4922 \mathrm{~dB}$ are obtained from simulation. In addition to it, reflection coefficient has been reduced to 0.4208 at the driven element. Table 2 shows the length and spacing for the 10 parasitic element array obtained from simulation.

Table 2: Length and spacing for the circular array element

\begin{tabular}{cccc}
\hline \multicolumn{2}{c}{ Length (1) } & \multicolumn{2}{c}{ Spacing(d) } \\
\hline 11 & $0.6681 \lambda$ & $\mathrm{d} 1$ & $0.1923 \lambda$ \\
12 & $0.6915 \lambda$ & $\mathrm{d} 2$ & $0.3249 \lambda$ \\
13 & $0.6505 \lambda$ & $\mathrm{d} 3$ & $0.2972 \lambda$ \\
14 & $0.6416 \lambda$ & $\mathrm{d} 4$ & $0.2902 \lambda$ \\
15 & $0.6337 \lambda$ & $\mathrm{d} 5$ & $0.5109 \lambda$ \\
16 & $0.5305 \lambda$ & $\mathrm{d} 6$ & $0.2305 \lambda$ \\
17 & $0.5399 \lambda$ & $\mathrm{d} 7$ & $0.2650 \lambda$ \\
18 & $0.5390 \lambda$ & $\mathrm{d} 8$ & $0.3110 \lambda$ \\
19 & $0.6572 \lambda$ & $\mathrm{d} 9$ & $0.3722 \lambda$ \\
110 & $0.4072 \lambda$ & $\mathrm{d} 10$ & $0.1185 \lambda$ \\
\hline
\end{tabular}

Obtained values of side lobe level and directivity from both examples show that the objective of low side lobe levels with maximum directivity has been achieved and the error is less in obtained and desired values. In addition to it, a better match between antenna and feed network is provided by minimizing the reflection coefficient of the driven antenna element. Obtained values of reflection coefficient from simulation for both examples show that matching is well under control for the driven antenna element.

Obtained results $-14.35 \mathrm{~dB}$ and $-15.078 \mathrm{~dB}$ in case of side lobe level $(\mathrm{dB})$ are better than for both the examples from
$-9.811 \mathrm{~dB}$ and $-11.03 \mathrm{~dB}[4],-12 \mathrm{~dB}$ and $-15 \mathrm{~dB}$ [5], $-12.24 \mathrm{~dB}$ and $-13.95 \mathrm{~dB}$ [6], $-13 \mathrm{~dB}$ and $-14.93 \mathrm{~dB}$ [8]. In case of side lobe level and directivity both, obtained results are better in terms of side lobe level where obtained directivity is little less from $[7,10]$ as we know both are contradictory to each other.

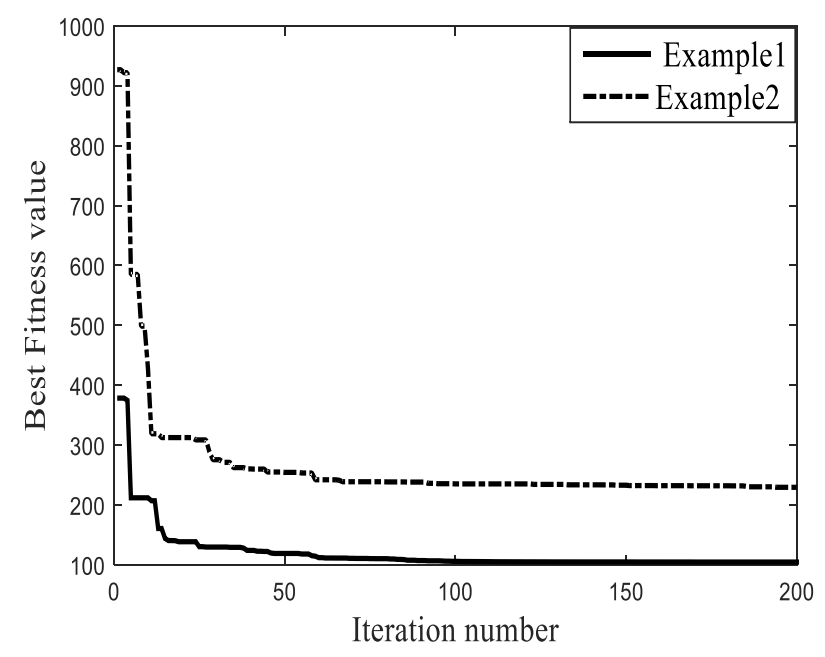

Fig. 5. Best fitness value versus iteration number.

Fig. 5 depicting the convergence of fitness value with the number of iterations. It gives a clear indication of the fitness value based on the fitness function with number of iterations.

\section{Conclusions}

This paper presents an alternative method for reduction of side lobe level with maximum directivity for circular antenna array including the mutual coupling effect instead of isotropic sources. Moreover, this technique is better than the previous techniques where excitation amplitudes are given to all the array elements. The use of single driven element provides a simple feeding network and hence this method is useful to reduce the complexity of the antenna. Two examples have been shown to illustrate the results obtained by this approach. Obtained results show the effectiveness of the proposed approach. This method works well for different antenna elements. In addition to it, reflection coefficient has been reduced to a great extent. This technique can be extended to other antenna array configurations including the ground plane effect also.

\section{References}

[1] R. S. Elliott, Antenna Theory and Design, Revised Edition. New Jersey: IEEE press, 2003

[2] M. I. Dessouky, H. A. Sharshar, Y. A. Albagory, "Efficient sidelobe reduction technique for small-sized concentric circular arrays" Prog. In Electromagn. Res., vol.65, 187-200, 2006.

[3] S.J. Blank and M. F. Hutt, "On the empirical optimization of antenna arrays" Antennas and Propagation Magazine, IEEE, vol.47 (2), 58-67, 2005. 
[4] M. A. Panduro, A.L. Mendez, R. Dominguez, G. Romero, "Design of non-uniform antenna arrays for side lobe reduction using the method of genetic algorithm" Int. J. Electron. Commun. (AEU), vol.60 (7), 713-717, 2006.

[5] M. Rattan, M. S. Patterh, B. S. Sohi, "Optimization of circular antenna arrays of isotropic radiators using simulated annealing" Int. J. Microw. Wirel. Technol., vol.1(5), 441-446, 2009.

[6] U. Singh, T. S. Kamal, "Design of non-uniformcircular antenna arrays using biogeography-based optimization" J. Microw. Antenna Propag., IET, vol.5 (11), 1365-1370, 2011.

[7] H. Wu, C. Liu, X. Xie, "Pattern Synthesis of Planar Nonuniform Circular Antenna Arrays Using a Chaotic Adaptive Invasive Weed Optimization Algorithm" Mathematical Problems in Engineering, vol. 2014, Article ID 575860, 1-13, 2014.

[8] G. Ram, D. Mandal, R. Kar, S. P. Ghoshal, "Circular and concentric circular antenna array synthesis using cat swarm optimization" IETE Technical Review, vol. 32 (3), 204-217, 2015.

[9] M. Shihab, Y. Najjar, N. Dib, M. Khodier, "Design of non-uniform circular antenna arrays using particle swarm optimization" Journal of Electrical Engineering, vol.59 (4), 216-220, 2008.

[10] P. Ghosh, J. Banerjee, S. Das, S. S. Chaudhury, "Design of non-uniform circular antenna arrays an evolutionary algorithm based approach" Progress In Electromagnetics Research B, vol. 43, 333-354, 2012.

[11] V. S. S. S. C. Vedula, S. R. C. Paladuga, M. R. Prithvi, "Synthesis of circular array antenna for side lobe level and aperture size control using flower pollination algorithm" International Journal of Antennas and Propagation, vol. 2015, Article ID 819712, 1-9, 2015.

[12] J. A. Rodriguez, A. Trastoy, J. C. Bregains, F. Ares, G. Franceschetti, "Beam reconfiguration of linear arrays using parasitic elements" Electron. Lett, vol. 42(3), 131-133, 2006.

[13] J. A. Rodriguez-Gonzalez, F. Ares-Pena, "Design of planar arrays composed by an active dipole above a ground plane with parasitic elements" Progress In Electromagnetics Research, vol.119, 265-277, 2011.

[14]R. F. Harrington, "Origin and development of the Method of Moments for field computation" IEEE Antennas and Propagation Mag., 32(3), 31-35, 1990.

[15] R.F. Harrington, "Matrix methods for field problems" Proc. IEEE, 55 (2), 136-149, 1967.

[16]S. J. Orfanidis, Electromagnetic waves and antennas, Ch. 21, 2003 www.ece.rutgers.edu/ orfanidi/ewa

[17] J. Sun, W. Fang, X. Wu, V. Palade, W. Xu, "Quantumbehaved particle swarm optimization: analysis of individual particle behavior and parameter selection" Evolutionary Computation, vol. 20(3), 349-393, 2012.

[18] J. Sun, W. Fang, V. Palade, X. Wu, W. Xu, "Quantum behaved particle swarm optimization with Gaussian distributed local attractor point" J. Appl. Math. Comput., vol. 218, 3763-3775, 2011.
[19]R. Muralidharan, A. Vallavaraj, G.K. Mahanti, A. Mahanti, "QPSO versus BSA for failure correction of linear array of mutually coupled parallel dipole antennas with fixed side lobe level and VSWR", Advances in Electrical Engineering, volume 2014, 1-7, Article ID 858290, 2014.

[20]H. Patidar, G.K. Mahanti, R. Muralidharan "Quantum particle swarm optimization for synthesis of nonuniformly spaced linear arrays with broadband frequency invariant pattern", Journal of Microwaves, Optoelectronics and Electromagnetic Applications, vol. 16 (3), 602-614, 2017.

[21]H. Patidar, G.K. Mahanti, R. Muralidharan "Synthesis of non-uniformly spaced linear array of unequal length parallel dipole antennas for impedance matching using QPSO”, International Journal of Microwave and Optical Technology, vol.12(3), 172-181, 2017.

[22] H. Patidar, and G.K.Mahanti, "Comparison of evolutionary algorithms for synthesis of non-uniformly spaced linear array of unequal length parallel dipole antennas for impedance matching with low side lobe level" Journal of Telecommunication, Electronic and Computer Engineering, vol.9(3), 121-127, 2017. 